\title{
Design and Implementation of a Compact Multifunctional Testing System for Standard Electric Energy Metering instrument with loading transmission line
}

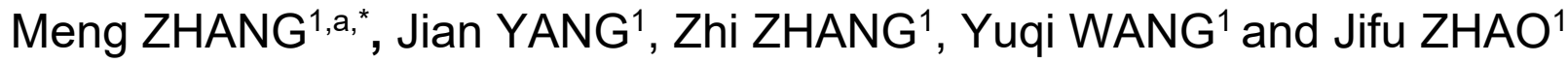 \\ ${ }^{1}$ State Grid Shandong Electric Power Research Institute, Jinan, Shandong, 250003, P. R. China \\ amengzhangxjtu@126.com
}

Keywords: Multifunctional testing, Energy metering, Automatic system

\begin{abstract}
This paper presents an intelligent test system for automated testing of energy metering standard devices. The energy metering standard devices in this paper refers specifically to the low voltage current transformer. Under the same current ratio, the device has the same appearance size, connection mode and capacity parameters, which facilitates the standardization of automated testing. The handling and wiring of a large number of standard devices usually require a lot of human resources, occupy space, and make the test inefficient. We designed a smart transmission line to realize the automatic handling. Meanwhile,the automatic connection of the electrical line is applied through the mechatronic module. The system also has good compatibility. In addition to routine testing, it is possible to implement a full range of test indicators such as limit temperature test, stress test, and residual magnetic measurement through a compatible muti-interface design.
\end{abstract}

\section{Introduction}

With the rapid development of China's power industry, as one of the important components of energy metering devices, the use of metering transformers has also increased [1]. Metering transformers must pass the qualified inspection of the tripartite institutions before they can be put into use. This increases the amount of testing tasks. Low-voltage current transformers have a large number of installations and rotations per year [2]. A medium-sized province needs hundreds of thousands of annual quantities every year. Completely relying on manual completion of complex testing processes is an almost impossible task. Therefore, after a unified manufacturing standard for such products and the standardization of sizes and specifications, large-scale automated assembly line testing techniques have emerged.

The assembly line is suitable for large-scale transformer testing. The testing speed is high and efficient. However, it requires a lot of space and space, and requires high construction cost. For small scaled sampling and full performance testing, the automated line system takes too long to transport the metering device [3]. It also occupies the normal running time of the assembly line, which leads to a decrease in overall efficiency [4]. The use of a traditional inspection station to perform sampling tasks is faced with a primary loop and a secondary loop requiring manual wiring. It is necessary to re-increase operating personnel and take time and effort.

Therefore, this paper presents a compact automated testing system that is suitable for small-scale transformer performance testing [5]. The specially designed intelligent transmission line and automatic wiring device eliminate most of the manual operation, and the compact design only occupies a limited space [6]. At the same time, multiple compatible interfaces can complete special test projects that cannot be applied by large-scale assembly line tests such as extreme temperature test,stress test, etc.

\section{System design and software platform structure}

In accordance with the design concepts of automation, the system uses the LMZ current transformer with a rated current of $75 \mathrm{~A}$ to $1500 \mathrm{~A}$ as the detection target. The system can complete multiple test modules including insulation resistance measurement, power frequency withstand voltage test, 
dielectric strength test, error measurement, and magnetic saturation measurement. Divided from the structure, the system consists of the main frame, automatic wiring module, test electrical equipment module, material transmission module and information identification module. The structure design of the compact muti-function testing system is shown in Fig. 1.

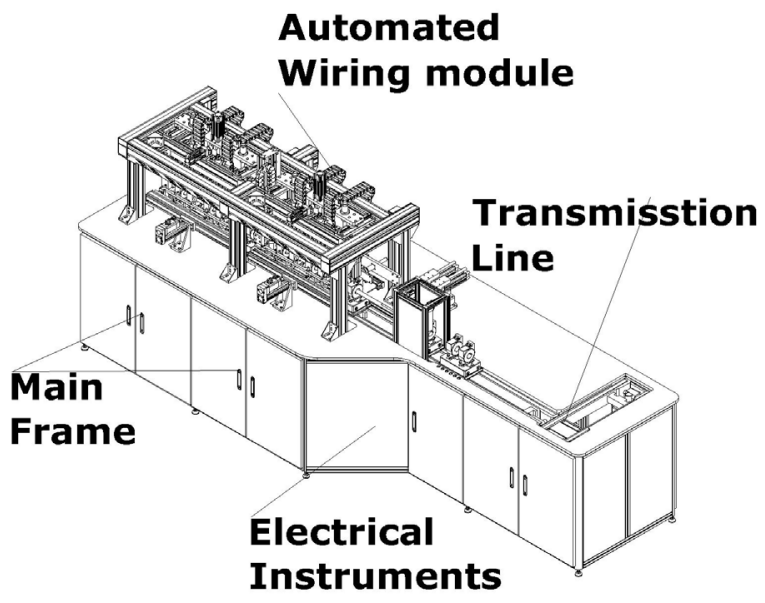

Fig. 1. The structure of compact muti-function testing system.

The software platform is the soul of automated inspection and delivery systems. The realization of the testing task depends on the effective cooperation between the software platform and the verification system. Therefore, the design needs to be rigorous, and the safety and reliability are high. In the design, the principles of standardization, openness, convenient management and maintenance, and information security are followed. The software platform consists of management, control, and device layers. The management team is a system management platform that accepts the testing plans issued by the production dispatching platform to manage and control the entire system, and reports the results and post-binding information to the production scheduling platform. The control layer is the conveying unit and completes the conveyance and positioning of the transformer during the testing process. The equipment layer is composed of several functional subsystems and executes the system management platform instructions to realize automatic testing of transformers except labeling.

\section{Main functional units design}

\subsection{Transmission line unit}

The transmission line system actually consists of two parts, the conveyor line body and the tooling tray of the fixed transformer. Its function is to realize the automatic circulation of multiple transformers on the test bench, eliminating the need for manual labor. Transmission line design using L-shaped structure, as shown in Fig. 2. Among them, the left-side vertical short body is used for the buffer of the tray tooling, and the long horizontal linear body is used for the flow of the transformer on the detecting table body, and the information is bound through the bar-code scanning and reading device in the middle.

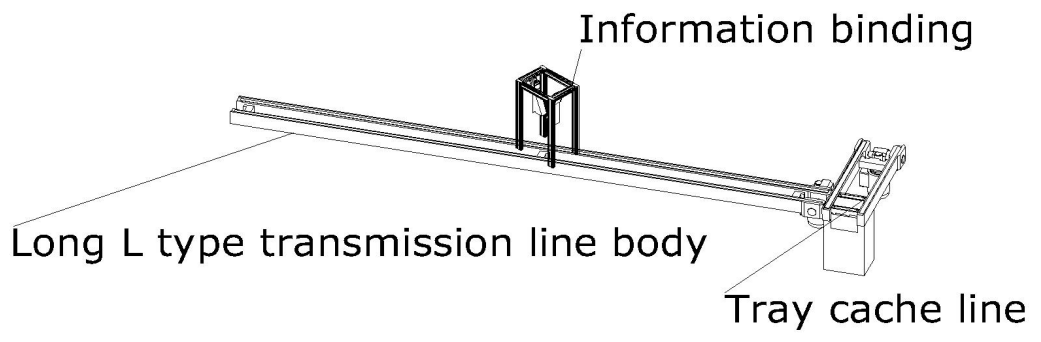

Fig. 2. The schema of L shaped transmission line system. 


\subsection{Automatic wiring mechanism}

The automatic wiring devices includes primary circuit connection/disconnection part and secondary circuit connection/disconnection part. The transformers tested in this article have adopted a through-type structure. The core of a wiring unit is to design the mechanism for automatically penetrating the copper rod. Usually, the through-cardiac structure should be placed beside the detection unit in parallel and occupy a lot of space. This article adopts the method of "passive" penetration of the copper rod into the transformer. The copper rod is relatively fixed on the test bench body. Through the program control, the transformer penetrates the fixed copper rod to realize a wiring process. The structure of primary connection part is shown in Fig. 3.

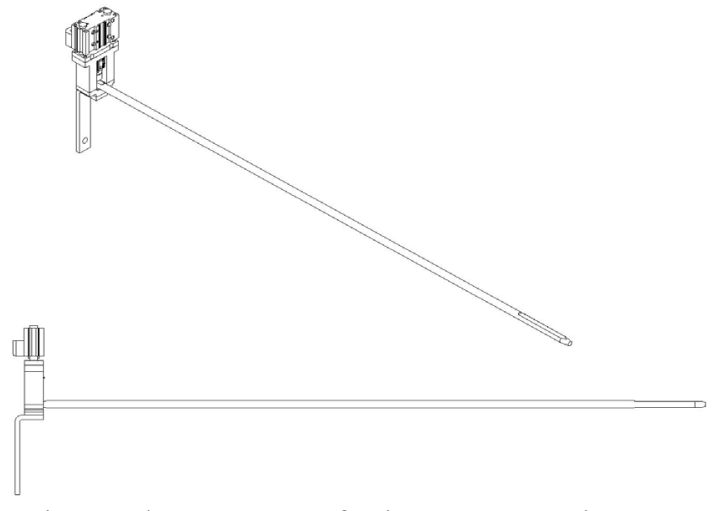

Fig. 3. The structure of primary connection part.

The transformer secondary wiring mechanism needs to consider four differences in the height direction, the largest deviation is $30 \mathrm{~mm}$. Since the transformer test is performed at the same station, it is required to design a universal secondary wiring mechanism. Secondary wiring devices include longitudinal electric cylinders, longitudinal guide shafts, transverse electric cylinders, transverse guides, and secondary wiring assemblies. When the device performs insulation resistance measurement, power frequency withstand voltage test and secondary winding inter-turn insulation strength test, magnetic saturation margin measurement and basic error measurement, the first wiring does not need to change, only the pressure wiring moves the cylinder to drive retraction, so that The rear secondary wiring assembly is aligned with the end of the transformer and the head of the transformer can be pressed. The integrate structure of secondary wiring mechanism is shown in Fig. 4.
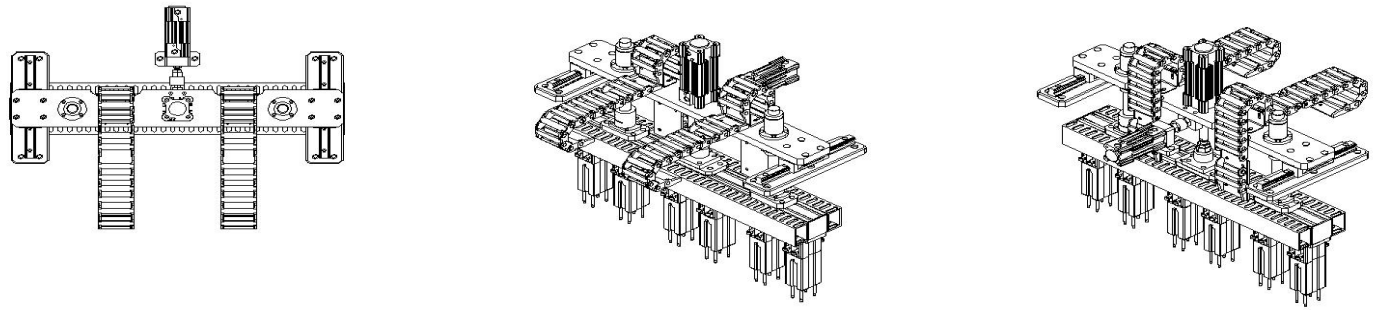

Fig. 4. The integrate structure of secondary wiring mechanism.

\section{Conclusions}

The new testing system seeks a balance between the fully automated system and the traditional artificial device to realize the optimization of economy and efficiency, and provides powerful technical support for the centralized testing center to realize intelligent, automated, and information-based work. Since the compact muti-function system was put into operation, it has been operating in a good state, meeting design goals and meeting the needs of production units. 


\section{References}

[1] G. Deng, X. Ma, and Z. Cao, Research and application of $0.4 \mathrm{kV}$ metering current transformers automatic verification system, Electrical Measurement \& Instrumentation, vol. 50(573), pp. 95-99, 2013.

[2] G. Dasilva, D. ILie, and S. Karnouskos, The impact of Smart Grid Prosumer Grouping on Forecasting Accuracy and Its Benefits for Local Electricity Market Trading, IEEE Transactions on Smart Grid, vol.5 (I.1), pp. 402-410, 2014.

[3] Z. Yan, Research and application of intelligent verification line system for electric energy meter, Electrical Measurement \& Instrumentation, vol. 46 (12), pp. 74-77, 2009.

[4] L. Gang, T. Lizhi, X. Qiong and H. Jiangning, Research on verification method of FA-200 transformers turn test sets calibrator, Northeast Electric Power Technology, vol. 11, pp. 9-11, 2014.

[5] L.H. Wang, and Y. H. Li, Risk Assessment and Risk Warning for Current Transformer Automatic Verification System, Applied Mechanics \& Materials, vol. 7, pp. 809-814, 2014.

[6] R. Wanjing, W. Baocai, and R. Zhongchen, Fault Analysis of Transformer Winding Turn Number Based on Ratio Test, Transformer, vol 49 (11), pp . 62-64, 2011. 